Supporting Information

\title{
Molecularly ordered ethylene-bridged periodic mesoporous organosilica spheres with tunable micrometer sizes
}

Yongde Xia, Zhuxian Yang, and Robert Mokaya*

School of Chemistry, University of Nottingham, Nottingham, NG7 2RD, UK 


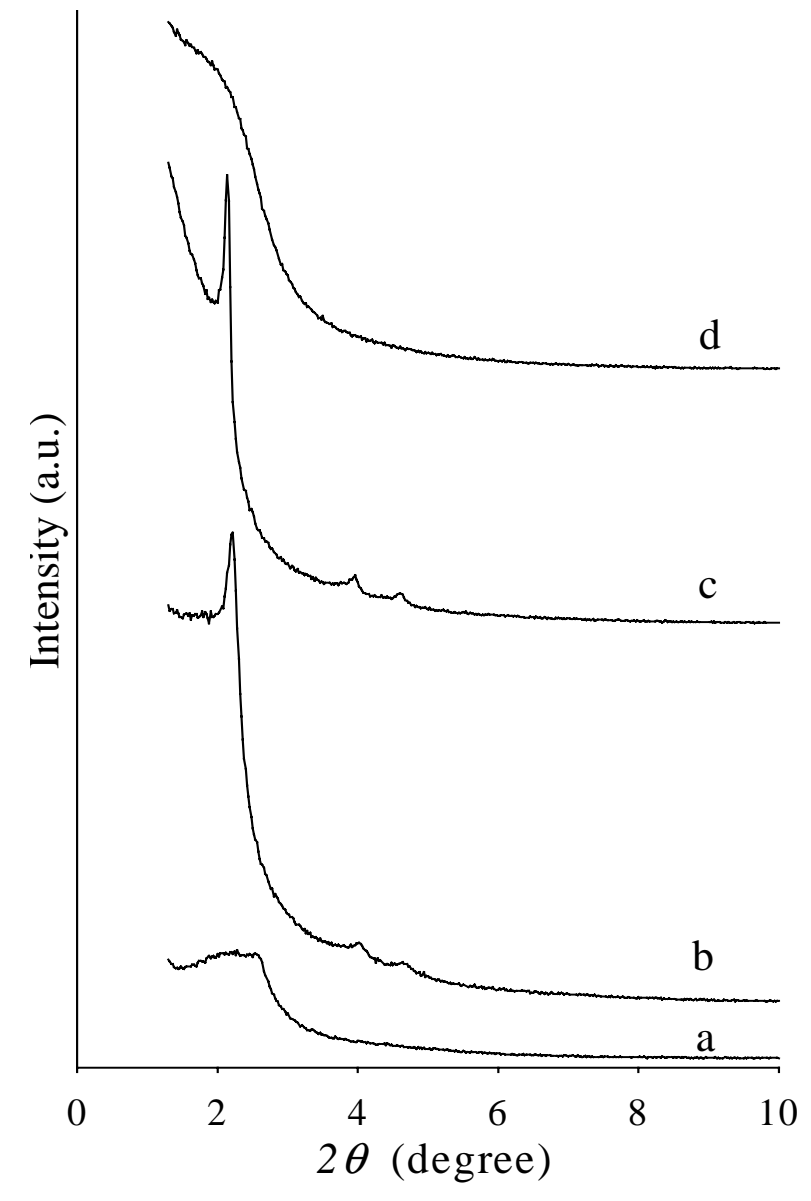

Supporting Figure 1S. Low angle region of powder XRD patterns of ethylene-bridged organosilica materials prepared at $\mathrm{H}_{2} \mathrm{O} / \mathrm{DTABr}$ molar ratio of 300 and varying basicity at $\mathrm{H}_{2} \mathrm{O} / \mathrm{NaOH}$ molar ratio of; (a) 225, (b) 200 , (c) 180 and (d) 163.6. The patterns show that the organosilica materials possess varying levels of mesostructural ordering. 


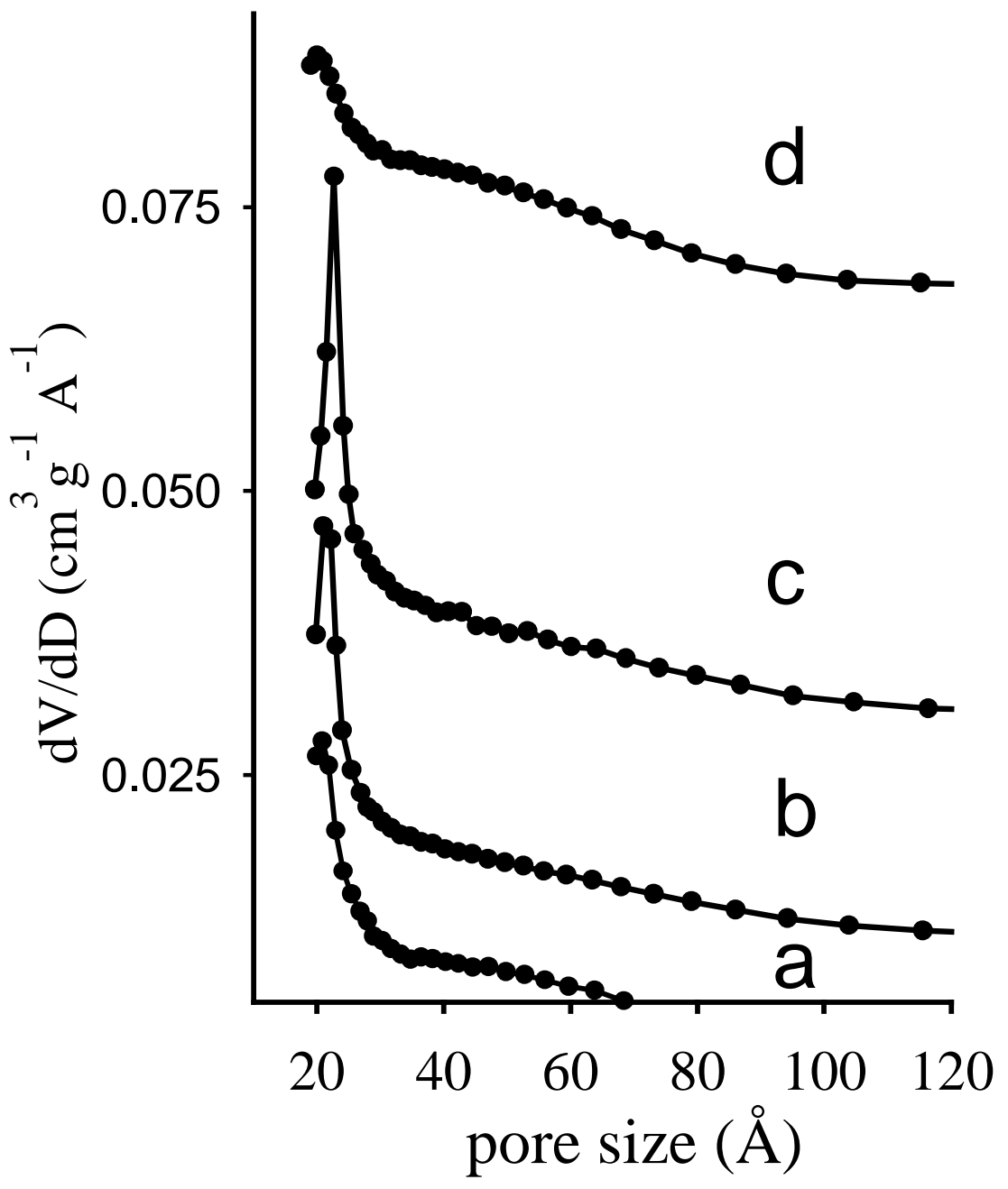

Supporting Figure 2S. Pore size distribution, over a wider pore size range, of ethylenebridged mesoporous organosilica materials prepared at $\mathrm{H}_{2} \mathrm{O} / \mathrm{DTABr}$ molar ratio of 300 and varying basicity at $\mathrm{H}_{2} \mathrm{O} / \mathrm{NaOH}$ molar ratio of; (a) 225, (b) 200, (c) 180 (d) 163.6 and (e) 150 . For clarity curves $b, c$ and $d$ are offset on the y-axis by $0.01,0.03$ and 0.07 respectively. The PSD curves confirm that the porosity of the PMOs is dominated by pores in the size range 20 $-25 \AA$, although there is also a contribution from pores in the range $40-60 \AA$. 

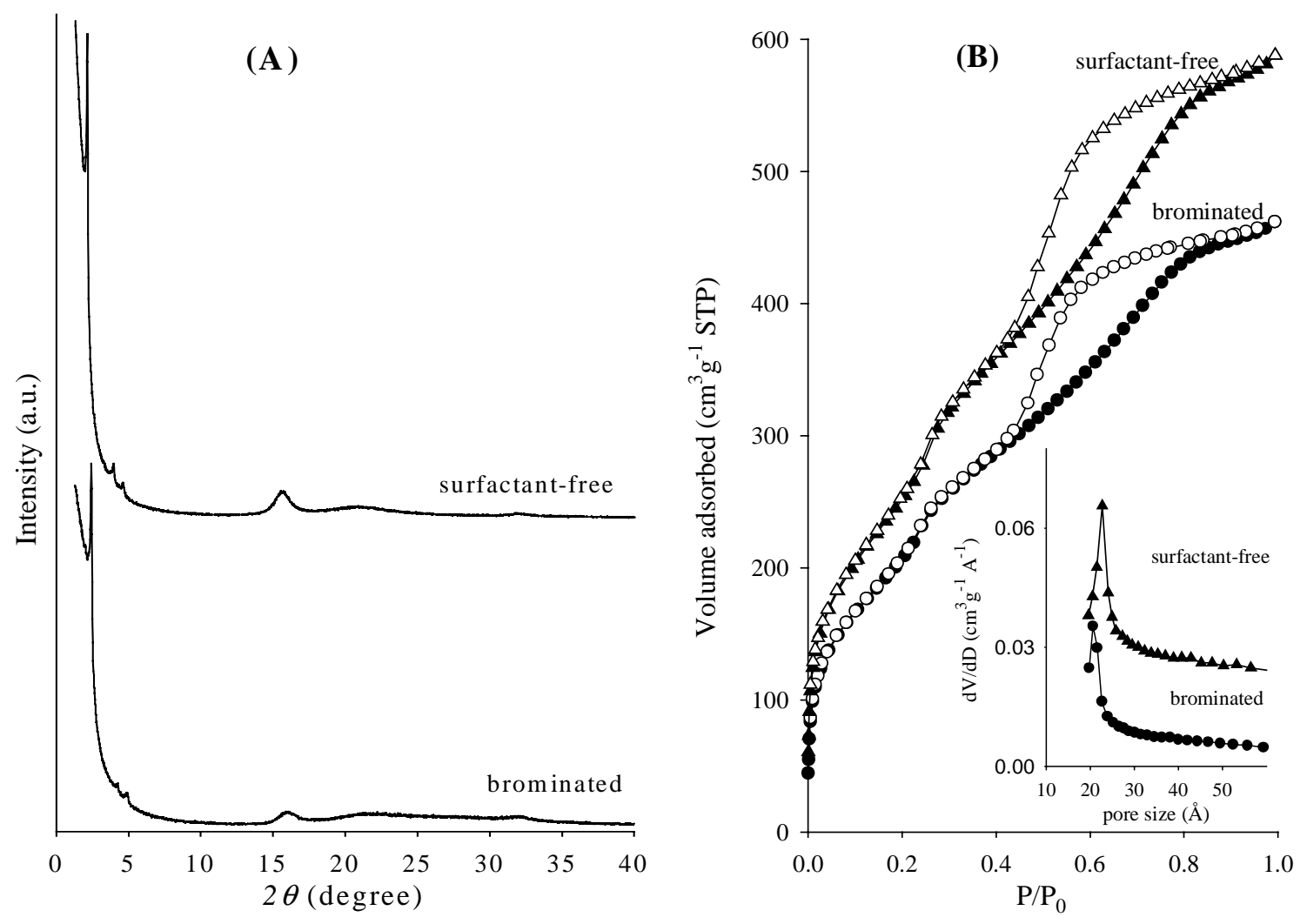

Supporting Figure 3S. Powder X-ray diffraction pattern (A) and nitrogen sorption isotherms (B) of spherical ethylene-bridged periodic mesoporous organosilica before and after bromination. 

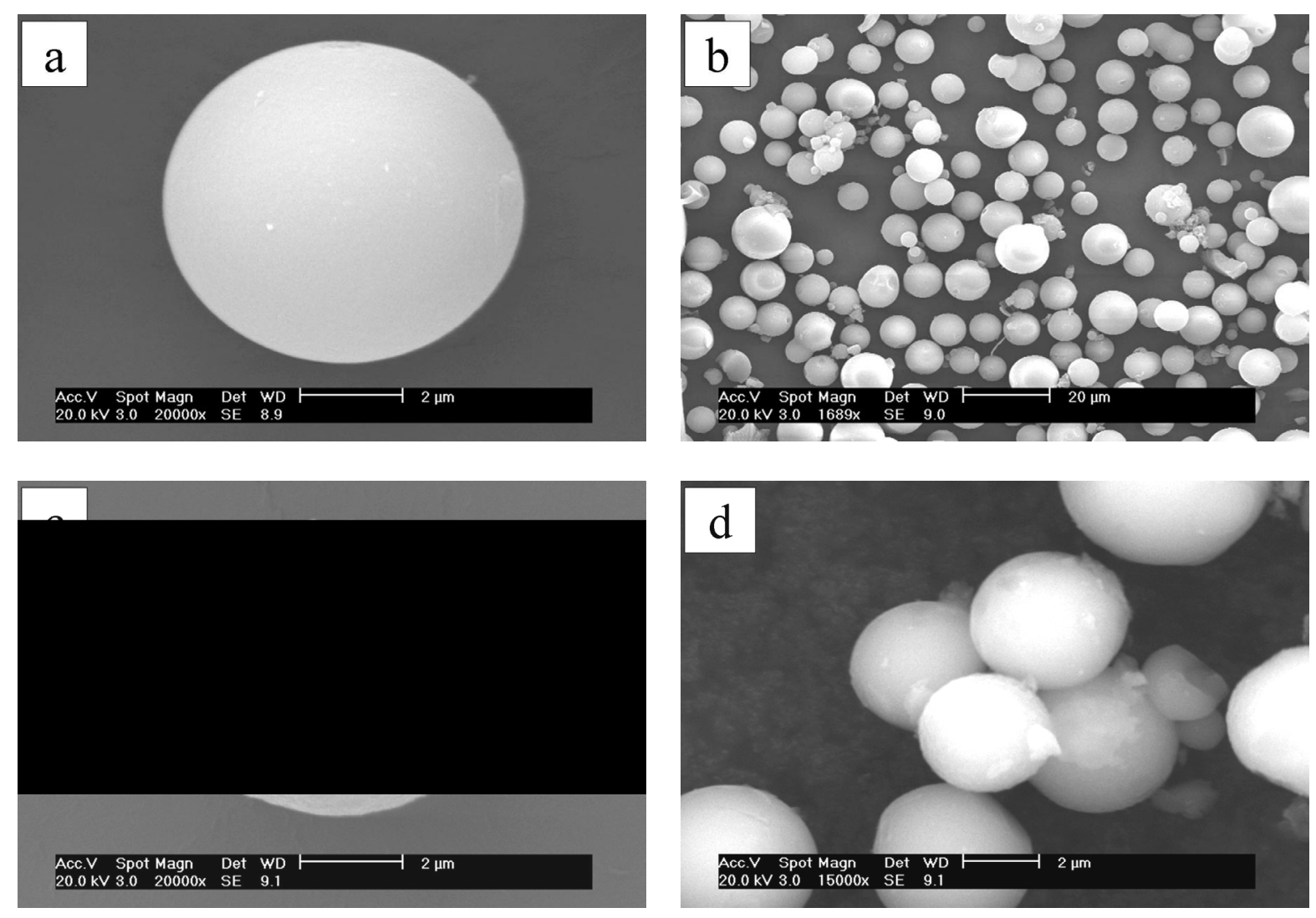

Supporting Figure 4S. Representative SEM images for ethylene-bridged mesoporous organosilica before $(\mathrm{a}, \mathrm{b})$ and after $(\mathrm{c}, \mathrm{d})$ bromination. The images show that bromination does not significantly affect the overall particle morphology but appears to increase the surface roughness of the spheres. 